\title{
The relationship between habitual anger coping style and serum lipid and lipoprotein concentrations
}

\author{
M.M. Müller* ${ }^{*}$, H. Rau ${ }^{b}$, S. Brody ${ }^{b}$, T. Elbert ${ }^{a}$, H. Heinle ${ }^{c}$ \\ ${ }^{a}$ Department of Psychology: University of Konstanz, Department of Psychology. P.O. Box 5560. \\ D-78434 Konstanz, Germany \\ ${ }^{b}$ Department of Medical Psychology, University of Tübingen, Tübingen, Germany \\ 'Physiological Institute, University of Tübingen. Tübingen, Germany
}

Received 14 March 1994; accepted 1 February 1995

\begin{abstract}
The relationship between habitual anger coping styles, especially anger expression in a socially assertive manner and serum lipid concentrations was examined in 86 healthy subjects. Habitual anger expression was measured by the Müller Anger Coping Questionnaire (MAQ) on four scales: Aggression, Controlled Affect, Guilt, and Social Inhibition. A positive correlation between serum cholesterol and Aggression was found, as was a negative correlation between LDL/HDL ratio and Controlled Affect. These correlations remained significant after adjustments for age, Body Mass Index (BMI), athletic activity, alcohol, nicotine and coffee consumption. After these adjustments, significant negative partial correlations between Controlled Affect and both total cholesterol and LDL were also found. We found no significant gender differences on any lipid measure. This study demonstrated that unfavourable lipid profiles are linked to a predominantly aggressive anger coping style, whereas favourable lipid profiles are associated with more socially assertive anger coping (Controlled Affect). Thus the expression of anger in a socially assertive manner can be seen as health promoting. The MAQ scales were better predictors of total serum cholesterol concentrations than the health related behaviors and characteristics. Results are discussed in terms of a psychophysiological risk model of inadequate anger coping.
\end{abstract}

Keywords: Descriptors: Anger; Habitual anger coping; Serum lipids; Cardiovascular diseases

Konstanzer Online-Publikations-System (KOPS)

URL: http://www.ub.uni-konstanz.de/kops/volltexte/2006/1906/ 


\section{Introduction}

The finding of a relationship of hostility and habitual anger coping to plasma lipid concentrations is of special interest in the field of behavioral medicine, since such a relationship would fit well with the finding that certain personality characteristics are related to cardiovascular diseases (see Müller, 1993a; Johnson, Gentry and Julius, 1992, for an extensive overview). Most of the previous research on the relationship of personality factors to cardiovascular diseases has been in investigating the relationship between hostility and plasma lipid concentrations, yet very little is yet known about whether specific habitual anger coping styles are related to unfavourable plasma lipid profiles. A closer inspection of the literature shows that the terms anger, hostility and aggression are often used interchangeably, an error also made by the lay public. Spielberger, Johnson, Russell, Crane, Jacobs and Wordan (1985) refered to these three concepts collectively as the AHA! syndrome (see also Johnson, 1990), where anger is at the core of the AHA! syndrome. Anger is an emotional state that varies in intensity and is neither a necessary nor a sufficient condition for the development of hostile attitudes and the manifestation of aggressive behavior (Johnson and Greene, 1992). Hostility as a very broad concept involves angry feelings and in addition has the connotation of negative, destructive attitudes, which in many instances motivates aggressive behavior (Johnson and Greene, 1992). From this clarification, one can conclude that anger and the way one copes with it seems to be the underlying dimension of hostility and aggression since anger can lead to hostile and aggressive behavior. Bearing this in mind, it is surprising that anger and anger coping has been nearly completely ignored in research investigating plasma lipid and lipoprotein concentrations as dependent variables.

As mentioned above, the hostility concept is a very broad one with many subcomponents (Buss and Durkee, 1957; Dujovne and Houston, 1991). Although a great deal of research has been done in the last decade (reviewed by Niaura, Stoney and Herbert, 1992), the results have been rather inconsistent, probably as a consequence of the multidimensional construct of hostility, the somewhat questionable validity of the Cook-Medley Hostility Scale (Cook \& Medley, 1954), which has been used in many studies ${ }^{1}$, and the incompatibility of other scales used in this field (e.g. the paranoid ideation subscale of the SCL-90 in Weidner, Sexton, McLellarn, Conner, \& Matatazzo, 1987; or the Bedford-Foulds Questionnaire in Fowkes, Leng, Donnan, \& Riemersma, 1992, see below).

\footnotetext{
${ }^{1}$ The Ho-Scale was based on Minnesota Multiphasic Personality Inventory (MMPI) items and was validated on violent adult convicts, suicidal outpatients and a group of graduate students with teaching experience. The generalizability to other populations has been questioned (Swan, Carmelli, \& Rosenman, 1990). Ho-Scale scores are linked with neuroticism, mistrust, cynicism and general psychopathology (Costa, Zonderman, McCrae, \& Williams, 1986; Megargee, 1985; Smith, Allred, \& Frohm, 1988; Swan et al., 1990). Although there are some reports on the reasonable construct validity of the Ho-Scale, a closer inspection shows that $\mathrm{Ho}$ was correlated with anger and anxiety in highly hostile men while interacting with their spouses and there was only a weak relationship between Ho-scores and hostile behavioral responsiveness during discussion for women (Smith, Sanders, \& Alexander, 1990). Pope, Smith and Rhodewald (1990) also interpreted their results as 'additional support for the construct validity of the Cook and Medley Ho scale' (page 511), but they used a rather artificial paradigm to test the construct validity and assessed the hostility related cognitions by means of self-report data. In both studies there
} 
Several studies have found a statistically significant relationship between hostility and both plasma total and LDL cholesterol (e.g. Dujovne \& Houston, 1991; Lundberg, Hedman, Melin, \& Frankenhaeuser, 1989; Rosenman \& Friedman, 1974; Siegler, Peterson, Barefoot, \& Williams, 1992; Weidner et al., 1987). The finding of higher plasma and LDL cholesterol in hostile subjects was replicated in a one year follow-up in the Portland Family Heart Study (Weidner et al., 1987), and remained significant after adjustments for age and body mass index. However, Fowkes et al. (1992) reported no relationships between serum total, HDL or LDL cholesterol and extrapunitivness, which was defined by hostile thoughts and denigration of others. In their sample of 1592 subjects in Edinburgh, they only found a statistically significant correlation between extrapunitive personality characteristics and serum triglyceride concentrations. This correlation remained statistically significant after adjustments for age, sex, smoking, alcohol consumption, and the other lipid concentrations. In addition to the Fowkes et al. study, Scherwitz, Perkins, Chesney, Hughes et al. (1992) also reported no relationship between hostility (as measured by the Cook-Medley Hostility Scale) and plasma cholesterol levels in the CARDIA study of 5115 subjects. In contrast to Fowkes et al. (1992), Dujovne and Houston (1991) and Weidner et al. (1987) found no significant relationship of plasma triglyceride concentrations to hostility.

In summary, the literature is controversial at best. One may keep in mind that the positive findings of epidemiological studies with respect to hostility are based on very small effect sizes as can be shown in the Fowkes et al. (1992) study, where the correlations accounted for much less than $2 \%$ of the variance, or the study of Siegler et al. (1992), in which a correlation between hostility scores and lipid ratios of 0.092 (controlled for age and sex) was highly statistically significant, but accounted only for $0.008 \%$ of the variance. One could conclude that the broad hostility concept is a predictor of questionable validity for plasma lipid and lipoprotein concentrations.

As has been argued above, anger and its expression seems to be the core concept of the so called AHA! syndrom. Previously, Johnson, Collier, Nazzaro and Gilbert (1992) showed that trait anger (the reported frequency of experienced feelings of anger, as assessed by the State-Trait Personality Inventory) was significantly related to the $\mathrm{LDL} / \mathrm{HDL}$ ratio among black males $(r=0.41)$. With respect to anger coping, Waldstein, Manuck, Bachen, Muldoon, \& Bricker (1990) reported a significantly positive correlation of anger-out as measured with Spielberger's (1988) State-Trait Anger Expresion Inventory (STAXI; see below for a critical assessment) and HDL $(0.46)$ in a sample of 29 young white males (average age 24 years). These two studies demonstrate that there exists a relationship between anger (and its expression) and

is a lack of clear definitions of anger and aggression and also, mostly undergraduates were investigated, which limits the generalizability of the findings. In the study by Smith et al. (1990), the marital interactions were analysed on the basis of high levels of marital satisfaction, which possibly limits the validity of the validitation variable. However, the studies of Smith et al. and Pope et al. are quoted as supporting the construct validity of the Ho scale, but both studies come to somewhat different conclusions regarding what Ho measures. Smith et al. (1990) stated that 'the Ho scale can be interpreted as reflecting, at least in part, the broad personality dimensions of agreeableness versus antagonism' (page 706), whereas Pope et al. (1990) stated that 'the Ho-scale appeared to be associated with the broader personality dimension of neuroticism or negative affectivity' (page 512). 
lipids but unfortunately they do not allow one to draw general conclusions with respect to this relationship since Johnson et al. had exclusively black males as subjects, which raises the question of possible ethnic differences, and Waldstein et al. had just 29 young subjects. In addition it is not clear if the study was controlled for healthrelated behaviors and characteristics, since the study was reported as an abstract.

To the best of our knowledge, there exists no study which assessed the full range of habitual anger coping styles (aggression, socially assertive anger expression, feelings of guilt after anger expression and anger related cognitions) and their relation to serum lipids. The aim of the present study was to test the relationship between specific habitual anger coping styles and serum lipid concentrations. In our previous research on anger coping and cardiovascular diseases such as essential hypertension and cardiac stroke (see Müller 1993c for an overview), we found that these patients were less able to express anger in an assertive manner as compared to healthy controls, which we interpreted as a behavior deficit, rather than a dominant individual coping style. From these results we hypothesized that unfavourable plasma lipid profiles are strongly related to the inability to express anger in a socially assertive manner.

\section{Methods}

\subsection{Anger coping assessment}

Anger coping was assessed by the Müller Anger Coping Questionnaire (MAQ, Müller \& Revenstorf, 1991; Müller, 1993b \& d), which measures anger coping as a trait in four dimensions: Aggression ( 7 items), Controlled Affect (expression of anger in a socially assertive way; 7 items), Guilt (6 items), and Social Inhibition ${ }^{2}$ (more cognitively related aspects of anger suppression; 8 items). Subjects were instructed to respond to each item on a four point scale with regard to how they generally behave or think when they are angry or furious $(0=$ almost never; $3=$ almost always). Internal consistency of the MAQ scales (Cronbach's alpha) ranged from 0.72 to 0.80 (Müller and Revenstorf, 1991). On the basis of factor scores, two additional scales can be built: General Anger-out (GAO; sum of Aggression and Controlled Affect) and Anger-in with Guilt (In/G; sum of Guilt and Social Inhibition). Previous validation data on the MAQ showed that none of the scales was significantly related to social desirability, as measured by the German version of the Crowne and Marlowe (1960) social desirability scale written by Lück and Timaeus (1969; Müller, $1993 \mathrm{~b} \& \mathrm{~d}$ ). The GAO scale represents the broader anger-out concept of the MAQ (in contrast to the STAXI; Spielberger, 1988), namely the general ability to express anger. General Anger-out and Anger-in with Guilt are unrelated, which was also confirmed in this sample $(r=-0.1, p=0.29)$.

\footnotetext{
${ }^{2}$ Originally these four factors were extracted as independent factors (PCA, Varimax-rotation). However, in this sample (see section 2.1.), we found weak but significant correlations between Aggression and Controlled Affect ( $r=0.26, p<0.05$ ), ard Aggression and Social Inhibition $(r=0.25, p<0.05)$. There was also a significant correlation between Guilt and Social Inhibition $(r=0.35, p<0.001)$.
} 
In contrast to the most prominent anger-coping questionnaire - the STAXI, the MAQ is more specific in assessing the possible facets of anger coping. In Spielberger's STAXI, anger-out is measured by aggressive behavior against persons or objects. The STAXI ignores the fact that anger can also be expressed in a socially assertive manner, for example, by simply talking to people. In fact, a major point in anger training programs is to teach the participants how to express anger in a socially assertive way (e.g. Deffenbacher, Story, Stark, Hogg, \& Brandon, 1987; Deffenbacher, 1993). The MAQ is the only existing questionnaire which considers both aggressive and socially assertive expressions of anger. A further weak point of the STAXI is the failure to consider cognitive components of anger expression. The MAQ assesses cognitions related to anger expression with the Social Inhibition scale.

As has been shown by Wallbott and Scherer (1989), behaviors such as withdrawing from people, which are assessed with the anger-in scale of the STAXI, are rarely related to anger. Such behavior is more often related to depression and grief. In fact, we were not able to extract such a behavioral anger suppression factor out of the initial item pool (Müller and Revenstorf, 1991). A further important component of anger expression, namely feelings of guilt, is also not represented in the STAXI. As has been shown in many experiments, feelings of guilt are positively related to recovery time of blood pressure in situations in which subjects had the possibility to act against a frustrator (see Stemmler, 1993 and Müller, 1993b for an overview).

\subsection{Subjects and Variables}

This study involved the participation of 120 healthy volunteers $(73$ men) aged $22-53$ (mean $=31.4$, S.D. $=6$ ). The investigation was part of a longitudinal study which will not be reported in this paper. Exclusion criteria were cardiovascular, neurological or psychiatric disorders. All subjects received informed consent, and the project was approved by the University of Tübingen ethics committee. Venous blood $(10 \mathrm{ml})$ was taken in order to determine lipid levels about $30 \mathrm{~min}$ after a laboratory session including mental arithmetic tasks, relaxation periods and a baroreceptor stimulation session. Prior to the laboratory session, subjects completed a demographic and health status questionnaire, including items on weight, height and coffee consumption. After the laboratory session, subjects received a diary to keep daily records of the duration of athletic activity, and alcohol and nicotine consumption for a one week period ${ }^{3}$. Alcohol consumption was scored as one dose for each 0.51 of beer, 0.251 of wine or 0.011 of distilled spirits. Median diary values of alcohol and cigarette intake were used for analyses. Subjects were requested to refrain from caffeine, nicotine and meals prior to the experimental session. Total plasma cholesterol, HDL and LDL cholesterol were analyzed enzymatically by means of the CHOD-PAP monotest method with standard kits (Boehringer Mannheim GmbH, Germany). The LDL/HDL ratio was also analysed, because it provides a good esti-

\footnotetext{
${ }^{3}$ In addition, subjects received an automatic blood pressure device to keep daily blood pressure records. The explanation of the use of the diary, the blood pressure device and other topics lasted about $30 \mathrm{~min}$, after which venous blood was taken.
} 
mate of the joint effect of these opposing cholesterol-lipoprotein fractions and serves as a good predictor for coronary heart disease (Kannel, Castelli, \& Gordon, 1979).

To make all analyses comparable, we excluded all subjects with missing data (e.g. in the diary or in the health questionnaire), leaving 86 subjects ( $53 \mathrm{men}$ ). Seven women were taking oral contraceptives.

\section{Analysis}

Correlations between anger coping styles and lipids were calculated and transformed to Fisher's $Z$. Partial correlations were computed for each lipid measure to test the relation between a certain anger coping style, as measured by the MAQ after adjustments for age, cigarette smoking, athletic activity, alcohol and coffee consumption, Body Mass Index (BMI) and the respective remaining three MAQ scales. Multiple regressions were performed to evaluate the percentage of explained variance of MAQ scales, health-related behaviors and characteristics, and all variables assessed for each lipid measure, respectively. The influence of health-related behaviors and characteristics on the habitual anger coping (MAQ scales) was evaluated by testing the semi-partial correlation coefficients (Cohen, 1975).

\section{Results}

Table 1 represents the characteristics of the study sample with respect to the assessed variables.

Table 1

Subject characteristics of males and females

\begin{tabular}{lrrrr}
\hline & $\begin{array}{l}\text { Males } \\
(n=53)\end{array}$ & & \multicolumn{3}{c}{$\begin{array}{l}\text { Females } \\
(n=33)\end{array}$} \\
\hline BMI & 23.95 & $(2.92)$ & $21.52^{* * *}$ & $(3.00)$ \\
Age & 31.51 & $(5.98)$ & 30.00 & $(5.56)$ \\
Coffee consumption & 3.42 & $(2.34)$ & 3.42 & $(1.87)$ \\
Smoking (overall) & 9.28 & $(9.67)$ & 10.42 & $(9.73)$ \\
Percentage of smokers & 58.49 & & 69.70 & \\
Smoking (smokers only) & 15.87 & $(7.36)$ & 14.96 & $(8.12)$ \\
Athletic activity & 18.70 & $(21.44)$ & 19.72 & $(19.64)$ \\
Alcohol consumption & 12.94 & $(11.94)$ & $5.49^{* *}$ & $(6.78)$ \\
Aggression & 3.83 & $(2.49)$ & 4.85 & $(2.97)$ \\
Controlled Affect & 11.04 & $(4.02)$ & 12.24 & $(4.49)$ \\
Guilt & 6.25 & $(3.63)$ & 6.79 & $(2.70)$ \\
Social Inhibition & 5.74 & $(3.01)$ & 6.91 & $(3.65)$ \\
Cholesterol & 205.85 & $(38.88)$ & 200.58 & $(38.93)$ \\
LDL & 118.85 & $(34.65)$ & 115.58 & $(39.34)$ \\
HDL & 54.98 & $(12.22)$ & 57.61 & $(22.78)$ \\
LDL/HDL & 2.33 & $(1.05)$ & 2.78 & $(1.20)$ \\
\hline
\end{tabular}

Coffee consumption in average cups per day. Smoking is in median of one week. Athletic activity is average activity within one week in minutes. Alcohol consumption is in median values according to the following scores (one point for each 0.51 of beer, $0.25 \mathrm{I}$ of wine or $0.01 \mathrm{I}$ of distilled spirits). MAQ scales $=$ factor scores. Serum lipids are in $\mathrm{mg} / \mathrm{dl}$. Standard deviations are in parantheses. ${ }^{* * *} p<0.001$; $* * p<0.01$ 
We only found significant gender differences with respect to BMI and alcohol consumption. In addition there were relatively more female smokers than male smokers, whereas there was no difference in the number of cigarettes smoked. Most importantly, we found no significant differences with respect to total plasma cholesterol, LDL, HDL, the LDL/HDL fraction and any anger coping measurement. For this reason, statistical analyses were calculated for the entire group.

\subsection{Lipid profiles}

As can be seen in Table 2, total serum cholesterol was positively associated with the MAQ Aggression scale $(r=0.21, p<0.05)$, while the LDL/HDL ratio was negatively associated with Controlled Affect $(r=-0.22, p<0.05)$

After adjustments for age, BMI, athletic activity, coffee, alcohol, nicotine consumption, and the respective remaining three MAQ scales, the correlations reported above between Aggression and total cholesterol, and between the LDL/HDL ratio and Controlled Affect remained significant $\left(r_{\mathrm{part}}=0.28, r_{\mathrm{part}}=-0.24, p<0.05\right.$ for both). With respect to total cholesterol, the partial correlation with Controlled Affect reached significance $\left(r_{\text {part }}=-0.24, p<0.05\right)$. This was also true for LDL and Controlled Affect $\left(r_{\text {part }}=-0.23, p<0.05\right)$. Unfavourable values and ratios were associated with expressing anger in an unassertive manner which also includes expressing anger aggressively (see Table 2 ).

Lastly, we tested the predictive power of habitual anger coping and the healthrelated behaviors on lipid measures. Calculation of semi-partial correlation coefficients assessed whether the additional consideration of either anger coping or health-

Table 2

Correlations and partial correlations (in parentheses) between MAQ scales (correlations printed in bold are related to hyotheses tested), health-related behaviors and lipid measures

\begin{tabular}{lcccc}
\hline & Cholesterol & LDL & HDL & LDL/HDL \\
\hline Age & 0.04 & -0.06 & 0.13 & 0.00 \\
BMI & 0.09 & 0.10 & -0.18 & 0.11 \\
Coffee consumption & -0.06 & -0.12 & 0.08 & -0.10 \\
Smoking & -0.08 & 0.15 & $-0.31^{* *}$ & $0.27^{*}$ \\
Athletic activity & -0.02 & -0.17 & 0.11 & $-0.21^{*}$ \\
Alcohol consumption & 0.06 & 0.01 & 0.10 & -0.04 \\
Aggression & $\mathbf{0 . 2 1 * ( 0 . 2 8 ^ { * } )}$ & $\mathbf{0 . 1 1}(\mathbf{0 . 1 8 )}$ & $\mathbf{0 . 0 7}(\mathbf{0 . 0 1 )}$ & $\mathbf{0 . 0 8}(\mathbf{0 . 1 5 )}$ \\
Controlled Affect & $\mathbf{- 0 . 1 0 ( - 0 . 2 4 ^ { * } )}$ & $\mathbf{- 0 . 1 5 ( - 0 . 2 3 ^ { * } )}$ & $\mathbf{0 . 0 9}(\mathbf{0 . 0 4 )}$ & $\mathbf{- 0 . 2 2 ^ { * }}\left(\mathbf{- 0 . 2 4 ^ { * } )}\right.$ \\
Guilt & $-0.16(-0.16)$ & $-0.06(-0.13)$ & $-0.13(-0.05)$ & $0.02(-0.10)$ \\
Social Inhibition & $-0.11(-0.14)$ & $0.02(-0.02)$ & $-0.17(-0.16)$ & $0.19 \quad(0.17)$ \\
\hline
\end{tabular}

Note (1). Partial correlations were calculated for each lipid measure separately by partialling out health-related behaviors and the remaining three MAQ scales. Note (2). For descriptive purposes, we also calculated the correlations between age, health related behaviours and the MAQ scales Guilt and Social Inhibition. We found a negative correlation between smoking (including all subjects, with nonsmokers scored as zero) and HDL ( $r=-0.31, p<0.01)$ and a positive relation was found between smoking and the LDL/HDL ratio $(r=0.27, p<0.05)$. Athletic activity was negatively related to LDL/HDL $(r=-0.21$, $p<0.05$ ).

${ }^{*} p<0.05,{ }^{* *} p<0.01$. Alphas are not Bonferroni-Dunn corrected. 
related behaviors variables lead to a significantly higher portion of explained variance, or whether theses variables explain just a common portion of variance. Fig. 1 depicts the percentage of explained variance by the four MAQ scales, six healthrelated behaviors variables, all ten variables assessed in this study and significant semi-partials.

Multiple regressions, using the four MAQ scales as predictors, showed that in predicting total cholesterol concentrations, Aggression and Controlled Affect revealed significant standard regression coefficients $(\beta=0.3$ and $-0.23, t=2.60$ and 2.06 respectively, and $p<0.05$ for both). With respect to $\mathrm{LDL}$, Controlled Affect approached significance $(\beta=-0.22, t=1.87, p=0.06)$ and was the sole significant predictor of the LDL/HDL ratio $(\beta=-0.24, t=2.15, p<0.05)$. As can be seen in Fig. 1, the health-related behaviors variables proved to be very poor predictors of total serum cholesterol which was also true for LDL. In estimating HDL concentrations, the health-related behaviors variables turned out to be much better than the MAQ scales, as BMI and tobaco consumption had significant Betas $(\beta=-0.25$ and $-0.39, t=2.47$ and 3.46, $p<0.05,<0.001$, respectively). The positive Beta of alcohol consumption failed to achieve significance $(\beta=0.19, t=1.81, p=0.07)$. Although the total multiple regression equation was significant for the health-related

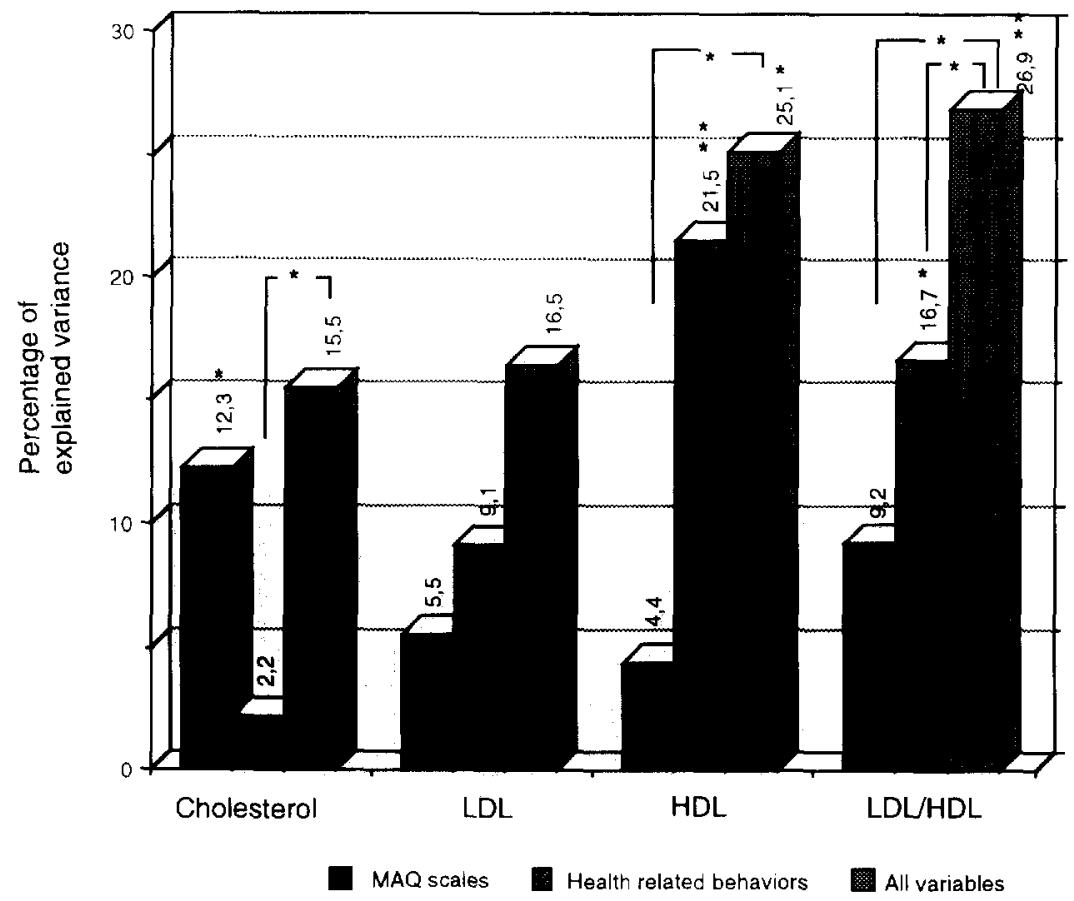

Fig. 1. Percentage of explained variance of serum total cholesterol, LDL, HDL and LDL/HDL ratio by MAQ scales, physiological variables and all variables together. Significant semi-partials are also shown. Note: ${ }^{*} p<0.05,{ }^{* *} p<0.01$. 
behaviors variables predicting the $\mathrm{LDL} / \mathrm{HDL}$ ratio, only smoking contributed significantly $(\beta=0.33, t=2.90, p<0.01)$. For each lipid measure, this profile remained the same when taking all variables into the equation.

The percentage of explained variance of total serum cholesterol increased significantly when adding the MAQ scales to the health-related behaviors variables $(F(4,76)=2.98, p<0.05)$, whereas no such effect could be found with respect to LDL. Predicting HDL showed a reverse pattern in which the MAQ scales were weak predictors, and variance explanation gained significantly by adding the healthrelated behaviors variables $(F(4,76)=3.49, p<0.05)$. Habitual anger coping and health-related behaviors and characteristics were both significantly poorer in explaining the variance of the LDL/HDL ratio when considered alone than when taken together $(F(4,76)=3.06$ and 2.64 respectively, $p<0.05$ for each).

\section{Discussion}

The primary focus of this study was to evaluate the relationship between serum lipid concentrations and habitual anger coping styles, especially socially assertive anger expression, by employing a more refined psychological measure than hostility with its many dimensions such as assault, indirect hostility, irritability, resentment, suspicion, verbal hostility and guilt (Buss and Durkee, 1957). The MAQ measures specific facets of habitual anger coping, namely Aggression, Controlled Affect, Guilt and Social Inhibition. We argued that the investigation of more specific personality characteristics (or more narrowly defined concepts) will bring us to a better understanding of possible psychophysiological mechanisms.

The results showed that the tendency to cope with anger in an aggressive manner was positively related to elevated total serum cholesterol and LDL/HDL ratio. None of the other variables paralleled these effects. It seems unlikely that other variables such as BMI, smoking, age, and alcohol consumption were responsible for the findings, which was confirmed by significant effects after adjustments were made for these variables.

With respect to expressing anger in a socially assertive manner (as measured by the Controlled Affect affect scale), negative correlations with serum cholesterol, $\mathrm{LDL}$ and the LDL/HDL ratio reached significance after adjustments for age, BMI, athletic activity, coffee, alcohol and nicotine consumption. In previous research, we demonstrated that patients suffering from essential hypertension and heart diseases were less able to express anger in an assertive manner than healthy controls (Müller \& Elbert, 1994) and chronic pain patients, which we interpreted as further evidence of a specific high risk profile for cardiovascular diseases with respect to anger coping (Müller, 1993c; Müller, Elbert, \& Rockstroh, 1993).

Anger is a very common emotional state, accompanied by increased autonomic activity, and there is some evidence of a predominantly noradrenergic influence (Stemmler, 1989, 1993; Müller, 1993b). Inadequate anger coping is related to prolonged recovery periods of the cardiovascular system, which can be interpreted as persistence of sympathetic activity (see Müller, 1993, for an overview). This autonomic reaction is mediated by the amygdala-sympathetic adrenal medullary sys- 
tem (Henry, 1986), confirmed by animal studies (e.g. Stock, Schlör, Heidt, \& Buss, 1978).

Although there are several possible mechanisms by which psychological factors such as personality characteristics produce changes in lipids and lipoproteins (as Niaura et al., 1992, have argued), the precise mechanism is still unknown. A good candidate for mediation is the sympathetic-adrenal-medullary system. Dimsdale, Herd and Hartley (1983) showed that infusion of epinephrine produces an increased level of cholesterol in animals, which may be the effect of the stimulation of adipose beta $_{2}$-receptors (see also Niaura et al., 1992). Norepinephrine may stimulate lipolysis by activating beta ${ }_{1}$-receptors in fatty tissue and/or by decreasing insulin levels (Freyschuss, Hjemdahl, Juhlin-Dannfelt, \& Linde, 1986; Hjemdahl \& Linde, 1983; Niaura et al., 1992). Fredrikson and Blumenthal (1992) recently showed that plasma norepinephrine during stress (mental arithmetic) correlated positively with triglycerides and the total to high density cholesterol ratio in healthy Type A men. However, they found no significant correlation between epinephrine and any lipid measure. Similar results with respect to sympathetic activation and higher lipid levels were reported by Fredrikson and Blumenthal (1988) and Suarez, Williams, Kuhn, Zimmerman and Schanberg, (1991).

As a psychophysiological model of the relationship between inadequate habitual anger coping and plasma lipid concentrations, it could be argued that people with poor anger expression skills frequently wish to express anger but are unable to do so competently. This skill deficit may result in a vicious circle in which they blame themselves for being aggressive or for not knowing how to react properly. This conflict may lead to prolonged (possibly noradrenergic) sympathetic activity, which in turn leads to elevations in total serum cholesterol, LDL and an unfavourable LDL/HDL ratio. The same mechanism (prolonged higher sympathetic activity) may also hold for the unfavourable lipid profiles of subjects with a predominantly aggressive anger coping style, but this possible link needs further exploration.

In former studies we (Müller \& Elbert, 1994; Müller, Elbert, Rau \& Zhuang, 1993; Müller, 1993c) identified the inability to express anger (which we see as a behavior deficit) as a more important risk factor than anger suppression. The present results support this contention, as there was no relationship between serum lipids or lipoproteins and the MAQ scales Guilt and Social Inhibition (which measure the tendency to suppress anger). If anger suppression is the dominant coping style of an individual, why should this be accompanied by increased autonomic activity, when the individual has learned that this coping style is successful in decreasing another's aggression? This argument was supported in a study by Engebretson, Matthews and Scheier (1989), where anger suppressors and anger expressors had the possibility to exhibit their preferred coping style. None of the groups showed prolonged blood pressure and heart rate recovery periods, whereas the 'pressure' (by experimental demand) to exhibit the nonpreferred, opposing coping style resulted in prolonged elevations of systolic blood pressure. Since the MAQ measures habitual anger coping style, subjects scoring high on anger suppression might have had positive experiences with their coping style. Somewhat surprising is the negative result with respect to Guilt, as one might have expected that feelings of guilt will also lead to an 
emotional state which might be accompanied by increased autonomic activity. One might speculate that guilt, like depression and helplessness, is more related to the activation of the hippocampus pituitary-adrenal cortical axis, which would lead to higher plasma cortisol levels (Henry, 1986).

To evaluate the postulated relationship between habitual anger coping styles, sympathetic nervous system activity, and lipids and lipoproteins, further research should consider additional biochemical variables such as catecholamines and cortisol. This would lead to a better understanding of which of the possible mechanisms are critical. In addition, the present study demonstrated that the additional assessment of physiological variables such as BMI, athletic activity, smoking, age and alcohol consumption will lead to a better portion of explained variance. This demonstrates again that the isolated consideration of either psychological or physiological variables will only give us a fractional understanding of the pathogenesis of psychosomatic diseases.

A second step would be to test whether these mechanisms are meaningful with respect to the incidence of cardiovascular diseases, which can only be evaluated in a longitudinal study. All of these possible studies would be well served by employing (or building upon) the advance in anger coping style measurement specificity presented in the current study. Summarizing our previous research on anger coping and cardiovascular diseases and the actual study we can draw the conclusion that expressing anger in a socially assertive manner seems to be health promoting whereas a lack of skills to do so is likely to be health damaging.

\section{Acknowledgement}

The study was supported by the Deutsche Forschungsgemeinschaft (El 101/3).

\section{References}

Buss, A.H., \& Durkee, A. (1957). An inventory for assessing different kinds of hostility. Journal of Consulting Psychology, 21, 343-349.

Cohen, J. (1975). Applied multiple regression/correlation analysis for the behavioral sciences. New York: Wiley.

Cook, W.W., \& Medley, D.M. (1954). Proposed hostility and PharisaicVirtue Scales for the MMPI. Journal of Applied Psychology, 38, 414-418.

Costa, P.T., Zonderman, A.B., McCrae, R.R., \& Williams Jr., R.B. (1986). Cynicism and paranoid alienation in the Cook and Medley HO Scale. Psychosomatic Medicine, 48, 283-285.

Crowne, D.P., \& Marlowe, D. (1960). A new scale of social desirability independent of psychopathology. Journal of Consulting Psychology, 24, 349-354.

Deffenbacher, J.L., Story, D.A., Stark, R.S., Hogg, J.A., \& Brandon, D.A. (1987). Cognitive-relaxation and social skills interventions in treatment of general anger. Journal of Counseling Psychology, 34, 171-176:

Deffenbacher, J.L. (1993). Grundlagen der therapeutischen Intervention bei Ärger. In: M.M. Müller (Ed.), Psychophysiologische Risikofaktoren bei Herz-Kreislauferkrankungen: Grundlagen und Therapie. Göttingen: Hogrefe Verlag.

Dimsdale, J.E., Herd, J.A., \& Hartley, L.H. (1983). Epinephrine mediated increase in plasma cholesterol. Psychosomatic Medicine, 45, 227-232. 
Dujovne, V.F., \& Houston, B.K. (1991). Hostility-related variables and plasma lipid levels. Journal of Behavioral Medicine, 14, 555-565.

Engebretson, T.O., Matthews, K.A., \& Scheier, M.F. (1989). Relations between anger expression and cardiovascular reactivity: reconciling inconsistent findings through a matching hypothesis. Journal of Personality and Social Psychology, 57, 513-521.

Fowkes, F.G.R., Leng, G.C., Donnan, P.T., Deary, 1.J., Riemersma, R.A., \& Housley, E. (1992). Serum cholesterol, triglycerides, and agression in the general population. Lancet, 340, 995-998

Fredrikson, M., \& Blumenthal, J.A. (1988). Lipids, catecholamines and cardiovascular responses to stress in patients recovering from myocardial infarction. Journal of Cordiopulmonary Rehabilitation, 12, 513-517.

Fredrikson, M., \& Blumenthal, J.A. (1992). Serum lipids, neuroendocrine and cardiovascular responses to stress in healthy type A men. Biological Psychology, 34, 45-58.

Freyschuss, U., Hjemdahl, P., Juhlin-Dannfelt, A., \& Linde, B. (1986). Cardiovascular and metabolic responses to low dose epinephrine infusion: an invasive study in humans. Clinical Science, 70 , 199-206.

Henry, J.P. (1986). Neuroendocrine patterns of emotional response. In R. Plutchik \& H. Kellerman (Eds.), Emotion (Theory, research, and experience, Volume 3). Orlando, San Diego: Academic Press Inc.

Hjemdahl, P., \& Linde, B. (1983). Influence of circulating NE and Epi on adipose tissue, vascular resistance and lipolysis in humans. American Journal of Physiology, 245, H447-H452.

Johnson, E.H. (1990). The deadly emotions. New York: Praeger.

Johnson, E.H., Collier, P., Nazzaro, P., \& Gilbert, D.C. (1992). Psychological and physiological predictors of lipids in black males. Journal of Behavioral Medicine, 15, 285-298.

In E.H. Johnson, W.D. Gentry \& S. Julius (Eds.) (1992). Personality, elevated blood pressure, and essential hypertension. Washington: Hemisphere Publishing Corporation.

Johnson, E.H., \& Greene, A.F. (1992). The interview method for assessing anger: development and validation. In E.H. Johnson, W.D. Gentry \& S. Julius (Eds.). Personality, elevated blood pressure, and essential hypertension. Washington: Hemisphere Publishing Corporation.

Kannel, W.B., Castelli, W.P., \& Gordon, T. (1979). Cholesterol in the prediction of atherosclerotic disease: New perspectives based on the Framingham Study. Annals of Internistic Medicine, 90, 85-91.

Lück, H.E., \& Timaeus, E. (1969). Skalen zur Messung Manifester Angst (MAS) uns sozialer Wünschbarkeit (SDS-E und SDS-CM). Diagnostica, 15, 134-141.

Lundberg, U., Hedman, M., Melin, B., \& Frankenhaeuser, M. (1989). Type A behavior in healthy males and females as related to physiological reactivity and blood lipids. Psychosomatic Medicine, 51, 113-122.

Megargee, E.I. (1985). The dynamics of aggression and their application to cardiovascular disorders. In M. Chesney \& R. Rosenman (Eds.), Anger and hositilty in cardiovascular and behavioral disorders (pp. 31-38). Washington, D.C.: Hemisphere.

In M.M. Müller (Ed.) (1993). Psychophysiologische Risikofaktoren bei Herz-Kreislauferkrankungen. Göttingen: Hogrefe.

Müller, M.M. (1993a). Das Müller Anger Questionnaire (MAQ): Konstruktion und Bestimmung der Gütekriterien. In M.M. Müller (Ed.), Psychophysiologische Risikofaktoren bei Herz-Kreislauferkrankungen: Grundlagen und Therapie. Göttingen: Hogrefe Verlag.

Müller, M.M. (1993b). Machen negative Emotionen krank? In M.M. Müller (Ed.): Psychophysiologische Risikofaktoren bei Herz-Kreislauferkrankungen: Grundlagen und Therapie. Göttingen: Hogrefe Verlag.

Müller, M.M. (1993c). Emotion und Herz-Kreislauferkrankungen. Psychophysiologische Determinanten von Ärger und Ärgerverarbeitung und deren Beitrag zur Pathogenese von essentieller Hypertonie. Psychological Institute, University of Tübingen, Tübingen: Unpublished dissertation.

Müller, M.M. (1993d). Fragebogen zur Erfassung des habituellen Ärgerausdrucks: Das Müller AngerCoping Questionnaire (MAQ). Zeitschrift für Diagnostische und Differentielle Psychologie, 14, 205-219.

Müller, M.M., \& Elbert, T. (1994). Ärgerverarbeitung bei kardiovaskulär Erkrankten. Psychotherapie, Psychosomatik, med. Psychologie, 44, 240-246. 
Müller, M.M. \& Revenstorf, D. (1991). Die Ärgerverarbeitungsskala. I. Itemanalyse und Reliabilitätsbetimmung. Psychotherapie, Psychosomatik, med. Psuchologie, 41, 138-146.

Müller, M.M.. Elbert. T.. \& Rockstroh. B. (1993). Ärgerverarbeitung bei kardiovaskulär Erkrankten. Verhaltensmedizin, 3 'suppl. 1), 56.

Müller, M.M. Elbert. T. Rau, H. \& Zhuang. P. (1993). Psychophysiologische Determinanten des habituellen Ausdrucks und deren Beitrag zur Chronifizierung von Bluthochdruck. Verhaltensmedizin. 3 / suppl. 1.39.

Niaura, R., Stoney, C.M. \& Herbert, P.N. (1992). Lipids in psychological research: the last decade. Biological Psychology, 34, 1-34.

Pope. M.K., Smith, T.W., Rhodewalt, F. (1990). Cognitive, behavioral and affective correlates of the Cook and Medley Hostility Scale. Journal of Personality Assessment. 54, 501-514.

Rosenman, R.H. \& Friedman. M. (1974). Neurogenic factors in pathogenesis of coronary heart disease. Medical Clinics of North America, 58, 269-279.

Siegler, I.C. Peterson, B.L., Barefoot, J.C.. \& Williams, R.B. (1992). Hostility during late adolescence predicts coronary risk factors at mid-life. American Journal of Epidemiology, 136, 146-154.

Scherwitz, L.W., Perkins, L.L., Chesney, M.A., Hughes, G.H., Sidney, S., \& Manolio, T.A. (1992). Hostility and health behaviors in young adults: the CARDIA study. American Journal of Epidemiology, 136, 136-145.

Smith, T.W., Allred, K.D., \& Frohm, K.D. (1988). Components of the Cook-Medley Ho scale. Proceedings of the 9th Annual Society od Behavioral Medicine (p. 155). Boston, MA.

Smith, T.W., Sanders, J.D. \& Alexander, J.F. (1990). What does the Cook and Medley Hostility Scale maesure? Affect, behavior, and attributions in the marital context. Journal of Personality and Social Psychology, 58, 699-708.

Spielberger, C.D. (1988). State-Trait Anger Expression Inventory. (Professional manual). Florida: Psychological Assessment Resources Inc.

Spielberger, C.D., Johnson, E.H., Russell, S.F., Crane. R., Jacobs, G.A. \& Worden. T.J. (1985). The experience and expression of anger: Construction and validation of an anger expression scale. In N.A. Chesney \& R.H. Rosenman (Eds.), Anger and hostility in cardioviscular and hehavioral disorders. New York: McGraw-Hill.

Vol 2 (pp. 159-187). Hillsdale. NJ : Erlbaum.

Stemmler, G. (1989). The autonomic differentiation of emotions revisted (Convergent and discriminant validation). Psychophysiology, 26, 617-632.

Stemmler. G. (1993). Peripherphysiologische Emotionsspezifität von Ärger. In M.M. Müller (Ed.), Psychophysiologische Risikofaktoren bei Herz-Kreislauferkrankungen: Grundlagen und Therapic. Göttingen: Hogrefe.

Stock, G.. Schlör, K.H., Heidt, H., \& Buss, J. (1978). Psychomotor behavior and cardiovascular patterns during stimulation of the amygdala. Pfluegers Archives, 376, 1:7-184.

Suarez, E.C., Williams, R.B., Kuhn, C.M., Zimmerman, E.H. \& Schanberg, S.M. (1991). Biobehavioral basis of coronary-prone behavior in middle-aged men. Parı II: Serum cholesterol, the Type A behavior pattern, and hostility as interactive modulators of physiological reactivity. Psychosomatic Medicine, $53,528-537$.

Swan, G., Carmelli, D., \& Rosenman. R.H. (1990), Cook and Medley hostility and the Type A behavior pattern: psychological correlates of two coronary prone behaviors. In M. Strube (Ed.), Type A behavior. (Special issue). Journal of Social Behavior and Personality. 5. 89-106.

Waldstein. S.R., Manuck. S.B., Bachen. E.A., Muldoon. M.F. \& Bricker, P.L. (1990). Anger expression, lipids, and lipoproteins. Poster presentation at the Eleventh Annual Meeting of the Society of Behavioral Medicine, Chicago, April 18-20, p. 102 (abstr.).

Wallbott, H.G., \& Scherer. K.R. (1989). Assessing emotion by questionnaire. In R. Plutchik \& H. Kellerman (Eds.), Emotion. theory, research and experience (Band 4). San Diego, New York: Academic Press.

Weidner, G., Sexton. G.. McLellarn, R., Conner. S.L., \& Matatazzo. J.D. (1987). The role of Type A behavior and hostility in an elevation of plasma lipids in adult women and men. Psychosomaric Medicine, 49, 136-145. 